Ann. Zootech., I964, 13 (I), 7I-85

\title{
INFLUENCE DU NUMÉRO DE LACTATION ET DU NIVEAU D'ÉTABLE SUR LA PRODUCTION LAITIÈRE DES VACHES MONTBÉLIARDES DU JURA
}

\author{
G. RICORDEAU, P. ALRIOL et J. LLFEBVRE \\ Avec la collaboration technique de M. Carpentier \\ Station centrale de Génétique animale, \\ Centre national de Recherches zootechniques, Jouy-en-Josas (Seine-et-Oise)
}

\section{SOMMAIRE}

L'objet de cette étude est de déterminer l'influence de différents facteurs - année, niveau des performances initiales, niveau d'étable - sur l'évolution de la production laitière avec l'âge.

De 1947 à 1958 , on a retenu dans 99 étables du Jura, I69 vaches Montbéliardes possédant les 5 premières lactations et 4663 lactations de " vaches contemporaines ". Pour chacune des étables, on a calculé + moyennes de production et un probit moyen.

I) De 1947 à $195^{8}$, on enregistre un accroissement continu de la production laitière moyenne dû en grande partie à l'amélioration des conditions de milieu : $30 \mathrm{~kg}$ par an pour les premières lacta* tions et $45 \mathrm{~kg}$ pour les lactations 2 à 5 (graph. I).

2) L accroissement de production dû réellement à l'influence de l'âge est le même pour l'échantillon des 169 vaches possédant 5 lactations consécutives que pour leurs contemporaines des mêmes étables, à condition d'éliminer les effets résultant de l'amélioration continue des conditions de milieu. Cet accroissement est en moyenne de $600 \mathrm{~kg}$ de lait de la première à la deuxième lactation, de $410 \mathrm{~kg}$ de la deuxième à la troisième, de $260 \mathrm{~kg}$ de la troisième à la quatrième et de $130 \mathrm{~kg}$ de la quatrième à la cinquième (tabl. 3). Les coefficients de répétabilité calculés à partir des données des I69 vaches avec 5 lactations sont de $0,5^{2}$ pour la quantité de lait et de 0,69 pour le taux butyreux.

3) Le " milieu étable " permet d'expliquer 25 à 30 p. Ioo de la variance des premières lacta. tions. Le coefficient de régression du niveau de production des génisses sur la moyenne d'étable à laquelle elles appartiennent est de 0,98 .

4) La production laitière moyenne des lactations 4 et 5 a été estimée à partir (tabl. 6) : de la production laitière en première lactation (équation $\mathrm{I}$ ); du niveau d'étable (équation 2 ) ; de ces deux variables (équation 3). Cependant, avec cette troisième équation, l'écart-type des erreurs entre la production adulte estimée et la production adulte réelle est encore de $579 \mathrm{~kg}$ de lait.

En valeur absolue, l'accroissement de production laitière avec l'âge est indépendant du niveau d'étable, mais à l'intérieur d'étables de même niveau, cet accroissement est en corrélation négative avec le niveau de production en première lactation.

5) Dans la discussion, il est envisagé de regrouper les étables en un petit nombre de classes pour estimer la "valeur d'élevage » des taurillons en testage à partir de la méthode dite " des contemporaines ". 


\section{INTRODUCTION ET BIBLIOGRAPHIE}

Quand il n'est pas possible d'utiliser exclusivement la moyenne des premières lactations pour caractériser le niveau de production d'une étable (effectifs trop petits), l'interprétation complète des résultats du testage nécessite souvent l'emploi de facteurs de correction permettant d'éliminer les effets de l'âge ou du numéro de lactation. En comparant les performances corrigées de chaque vache à la production moyenne du troupeau corrigée pour l'âge, les productions relatives ainsi obtenues peuvent être utilisées pour estimer la valeur d'élevage des taureaux dont les filles sont disséminées dans différentes catégories d'élevages.

De nombreuses études ont été consacrées au problème de l'influence du facteur âge (âge exact ou numéro de lactation) sur la production laitière, mais elles tiennent rarement compte du niveau de production de l'animal et du niveau d'étable auquel il appartient. Les auteurs ayant par ailleurs travaillé sur des races différentes, en utilisant des facteurs de correction additifs ou multiplicatifs, il ne faut pas s'étonner si les résultats obtenus sont parfois contradictoires.

\section{Niveau de production en $1^{\text {re }}$ lactation}

Si l'on se base uniquement sur le niveau de production en Ire lactation, l'accroissement (absolu ou relatif) de production avec l'âge est plus important pour les faibles productrices comme l'indiquent SANDERs (I928), WARD et CAMPBELL (I938), JohaNsson et Hansson (I940), LefFebvre (I946), Hickman et Henderson (I955), Poly (I958), Auriol et Ricordeau (I958), Pirchner (I959), bien que Bakels et Gali. (I96I), n'observent pas de différences et que DIETRICH (I957) et Brumby (I96I), font apparaître des résultats différents.

Du point de vue de la sélection, il y a lieu de souligner que si la relation existant entre le niveau de production des génisses et l'augmentation de production avec 1'âge est légèrement négative en ce qui concerne les corrélations phénotypiques, elle apparaît comme nulle en ce qui concerne les corrélations génétiques (HICkMAN et Henderson, i955; Robertson et Khishin, I958; Pirchner et Lush, i959).

\section{Performances obtenues sur plusieurs lactations}

Le niveau de production des génisses peut ne pas refléter parfaitement leurs performances ultérieures (AURIoL et GrosclaUde, ig60). Beaucoup de génisses sont handicapées par un vêlage intervenant précocement (ou en saison défavorable) ; elles subissent une augmentation anormale de la production laitière au cours des lactations suivantes. Il est alors possible de réduire cet inconvénient en tenant compte des performances enregistrées sur l'ensemble des lactations. En se basant sur la moyenne des 4 premières lactations, SANDERs, Johansson et Hansson, constatent de faibles différences dans l'augmentation de la production laitière avec l'âge : cette augmentation étant même supérieure pour le groupe des fortes productrices. 


\section{Moyenne d'étable}

La détermination du niveau de production à partir des performances enregistrées au cours de la vie d'un animal nous conduit à prendre en considération le niveau d'étable. Ce paramètre, estimé par une moyenne de production laitière, est influencé en grande partie par les conditions du milieu. En effet, pour ROBERTson et Rendel (I954), Robertson et McArthur (1955), Pirchner et Lush (I959), SyrSTAd (ig6o), Brumby (Ig60, ig6i), Wiener (ig60), Thompson, Baldwin et Graf (I96I), les différences génétiques n'expliquent que 4 à 25 p. Ioo de la variance des écarts entre élevages. Il existe donc une régression importante de la production des vaches sur la moyenne de production du troupeau.

\section{TABLEAU I}

Régression de la production laitière des vaches sur la moyenne de production de leur étable ou celle de leurs contemporaines

\begin{tabular}{|c|c|c|}
\hline Auteurs & $\begin{array}{l}\text { Coefficient } \\
\text { de régression }\end{array}$ & Ubservations \\
\hline ROBERTSON et RENDEL $(\mathbf{1 9 5} / 4)$ & $\begin{array}{l}1,08 \\
0,90 \\
0,91 \\
0,95\end{array}$ & $\begin{array}{l}\text { Frisonnes } \\
\text { Shorthorn } \\
\text { Guernesey } \\
\text { Toutes races }\end{array}$ \\
\hline HOFMEYR (1955) & 0,71 & Vaches Danoises et Suédoises, génisses d'I.A. \\
\hline IIENDERSON (1956) & 0,60 & $\begin{array}{l}\text { Génisses d'I. A.; moyenne du troupeau calculée d'après la } \\
\text { production des vaches adultes corrigée pour l'áge, ou } \\
\text { d'après la production des contemporaines de même àge. }\end{array}$ \\
\hline WiNTERs et ERB (1957) & 0,86 & $\begin{array}{l}\text { Vaches Guernesey, Frisonnes et Jersiaises; régression de la } \\
\text { production de matiere grasse des filles sur la production } \\
\text { moyenne des contemporaines. }\end{array}$ \\
\hline GaLnT et Goodwin (1958) & $\begin{array}{l}0,89 \text { à } 0,95 \\
0, \gamma^{\prime}+\text { d } \\
0,96\end{array}$ & $\begin{array}{l}\text { Frisonnes } \\
\text { Guernesey }\end{array} \begin{array}{c}\text { Régression intra-père de la production des filles } \\
\text { sur la moseme du troupeau corrigée ou non. }\end{array}$ \\
\hline SCHINDLER (1960) & 0,65 & \\
\hline ․ A. T. Suède $(1960)$ & $\begin{array}{l}0,85 \\
0,79\end{array}$ & $\left|\begin{array}{l}S R B \\
S L B\end{array}\right| 1^{\text {re }}$ lactation \\
\hline
\end{tabular}

Les résultats concernant l'augmentation de la production laitière avec le numéro de lactation, en fonction du niveau d'étable, ne concernent que les premières lactations et sont assez contradictoires. Pour Johansson et Robertson (I952), HickMAN et HENDERSON (I955), l'accroissement de production laitière avec l'âge est d'autant plus faible que le niveau d'étable est plus élevé. HOFMEYR (I955), WIENER (I960), SEARLE et HENDERSON (I959), observent le phénomène inverse, et cela, selon les deux derniers auteurs, indépendamment du niveau de production des génisses. 


\title{
Facteurs de correction
}

La recherche des facteurs de correction doit être entreprise avec prudence, si l'on veut comme le souligne JoHANSSON (I958), tenir compte des relations et des interactions existant entre les différents facteurs de variation. Si les études de JoHANsson et de Hansson (r940), Searle et Henderson (I960), Searle (I962) ne permettent pas de choisir entre facteurs additifs ou multiplicatifs, celles de VAN VLECK et Henderson ( $\mathrm{Ig} 6 \mathrm{I}$ ) ont mis en évidence la nécessité de calculer les facteurs de correction en considérant simultanément les variables liées entre elles.

\section{MATÉRIEI F,T TECHNIQUES}

\author{
Choix des données
}

Pour étudier l'évolution de la production laitière avec l'âge, nous avons retenu 99 étables comptant chacune au moins une vache ayant eu les cinq premières lactations contrôlées. Afin d'éliminer le facteur " saison de vêlage ", n'ont été retenues que les vaches ayant toujours vêlé pendant la saison la plus favorable à la production laitière, c'est-à-dire entre septembre et mars (AURIOL, I955). D'autre part, les animaux dont une lactation (ou plus) avait une durée inférieure à 240 jours ou supérieure à 370 jours, ont été éliminés de cette étude. 169 vaches ont ainsi été sélectionnées pour la période de 1947 à 1958.

\section{Calcul des moyennes d'étable}

Pour chacune des 99 étables précédentes, nous avons calculé 4 moyennes : moyennes des Ires, moyenne des $2^{\mathrm{e}}$, moyenne des $3^{\mathrm{e}}$ et $4^{\mathrm{e}}$, et moyenne des $5^{\mathrm{e}}$ lactations. Le calcul de ces moyennes a été effectué à partir des lactations comparables, obtenues de 1947 à 1958, des animaux contemporains appartenant à ces 99 étables : 4663 lactations correspondant à des vêlages en saison favorable et de même limite de durée ont ainsi été utilisées.

Chacune des 4 moyennes d'étable a été transformée en probit en fonction des moyennes respectives des lactations I, 2, 3-4 et 5. La moyenne pondérée des 4 probits donne le probit moyen d'étable ; le coefficient de répétabilité des 4 probits calculés pour chaque étable est de 0,75 .

\section{Corrections}

Les corrections utilisées sont celles établies antérieurement par AURIOL et RICORDEAU (1958) : les lactations supérieures à 300 jours ont été ramenées à cette durée en appliquant une régression journalière de $9 \mathrm{~kg}$ de lait pour les premières lactations, et de $\mathrm{I} 3 \mathrm{~kg}$ pour les lactations 2 à 5 ; l'âge au premier vêlage a été ramené à $3^{2}$ mois en appliquant une régression mensuelle de $68 \mathrm{~kg}$ de lait de 24 à 32 mois, aucune correction n'étant faite au-delà de 32 mois d'âge.

\section{Accroissement de production avec l'âge : $\mathrm{D}_{5-1}$}

Pour caractériser l'augmentation de production laitière avec l'âge des vaches possédant 5 lactations consécutives, nous avons calculé la différence $\mathrm{D}_{5-1}$ entre la production moyenne des $4^{\mathrm{e}}$ et $5^{\mathrm{e}}$ lactations et la production obtenue en $\mathrm{I}^{\mathrm{re}}$ lactation. Les corrections pour la durée de lactation ont été appliquées aux lactations I, 4 et 5 supérieures à 300 jours. 


\section{RÊSULTATS}

\section{I. - DiFFÉRENCES ANNUELL ES E'T DIFFÉRENCES ENTRE ÉLEVAGES}

\section{a) Différences annuelles}

A partir de 1'ensemble des données récoltées de I947 à I958 dans les 99 étables nous avons calculé les moyennes mobiles annuelles par numéro de lactation. Indé-
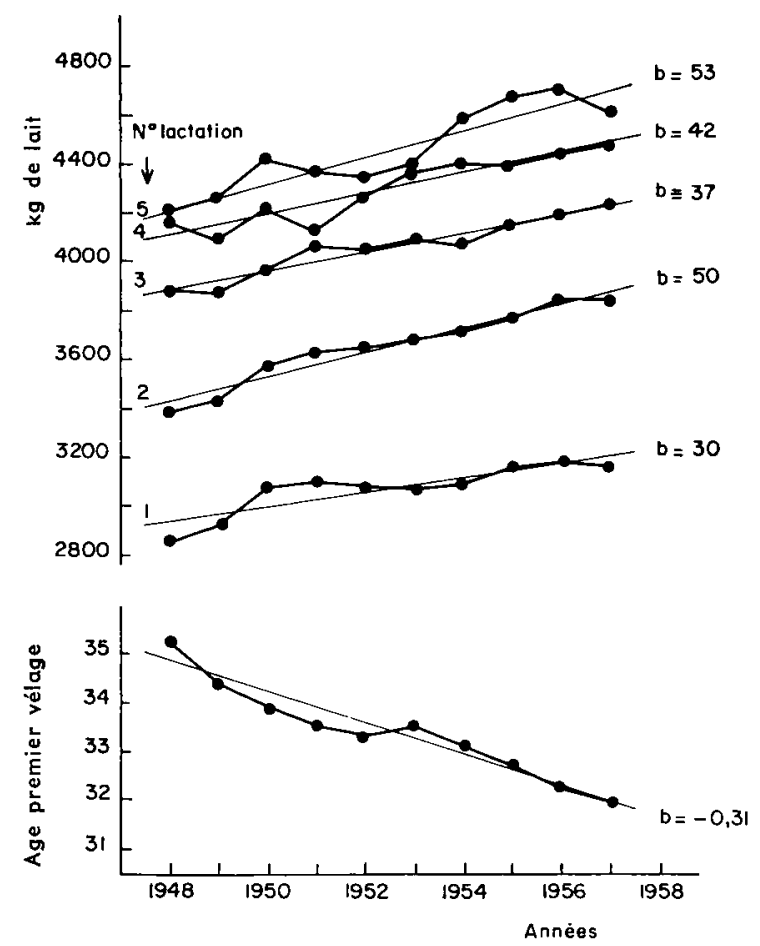

GRAPH. I. - Évolution de l'âge au premier vélage et de la production laitière moyenne sur onze années consécutives ( 1947 à 1958) en fonction du numéro d'ordre de la lactation.

$b$ : coefficients de régression de la production laitière annuelle pour les lactations I, 2, 3, 4 et 5 .

pendamment de l'âge des animaux, il apparaît un accroissement constant de la production laitière. Cette amélioration annuelle est en moyenne de $45 \mathrm{~kg}$ pour les lactations $2,3,4$ et 5 et de $30 \mathrm{~kg}$ seulement pour les premières lactations, vraisemblablement par suite d'une diminution de l'âge moyen au premier vêlage ( $\mathbf{r}$ mois tous les 3 ans en moyenne - graphique I). L'insémination artificielle est trop récente dans le Jura (I947) pour être responsable à elle seule de cette amélioration. Celle-ci serait donc largement attribuable aux conditions d'élevage. HOFMEYR (I958) signale qu'en Suède, au cours des Io dernières années (I945 à I955) la production de toutes les vaches contrôlées ( $27 \mathrm{p}$. Ioo du total) a augmenté d'environ $400 \mathrm{~kg}$ de lait. Ayant contrôlé 35 troupeaux sur 9 années, WIENER (I960) observe également une réduction 
des différences entre troupeaux due à l'augmentation du niveau des étables médiocres et non à la diminution du niveau des bons élevages.

\section{b) Différences entre élevages}

I a production laitière moyenne varie de façon importante d'un élevage à l'autre, puisque l'écart maximum entre élevages atteint I $620 \mathrm{~kg}$ pour les moyennes calculées d'après les premières lactations, et $2900 \mathrm{~kg}$ pour les moyennes calculées d'après les $5^{\mathrm{e}}$ lactations (tabl. 2). Le nombre moyen de lactations utilisé pour chaque moyenne d'élevage est respectivement de I3, I2, I6 et I3 pour les lactations I, 2, 3 et 4,5 (effectif légèrement plus élevé pour les bonnes étables), ce qui semble indiquer que dans les 99 élevages retenus, on a renouvelé régulièrement les troupeaux en élevant peu de génisses. Les différentes moyennes d'étables, y compris celles calculées d'après les premières lactations sont pratiquement indépendantes de l'âge moyen au premier vêlage de chacune des étables.

\section{TABIEAU 2}

Moyennes d'étables calculées sur une période de onze ans, en $\mathrm{kg}$ de lait (99 étables)

\begin{tabular}{|c|c|c|c|c|c|c|}
\hline $\begin{array}{c}\text { No } \\
\text { de lactation }\end{array}$ & Moyenne & $\begin{array}{l}\text { Écart-type } \\
\text { (coefficient } \\
\text { de variation) }\end{array}$ & $1^{\text {er }}$ quartile & $3^{\mathrm{e}}$ quartile & $\begin{array}{c}\text { Écart } \\
\text { maximum } \\
\text { entre } \\
\text { élevages }\end{array}$ & $\begin{array}{c}\text { Effectif } \\
\text { moyen } \\
\text { de lactations } \\
\text { par élevaure }\end{array}$ \\
\hline 1 & 3068 & $338(11,0 \%)$ & $28: 37$ & 3305 & 1620 & 13,1 \\
\hline 2 & 3693 & $462(12,5 \%)$ & $: 3: 350$ & 1025 & 2340 & $11, \pi$ \\
\hline 3 et 4 & 1766 & $462(11,1 \%)$ & 3800 & 4175 & 2250 & $16,4_{k}$ \\
\hline 5 & 4481 & $524(11,7 \%)$ & 4111 & 4809 & 2900 & 13,1 \\
\hline
\end{tabular}

\section{2. - ACCROISSEMENT DE PRODUCTION LAITIÈRE} EN FONCTION DU NUMÉRO DE LACTATION

Cet accroissement a été calculé pour les I69 vaches possédant les cinq premières lactations, mais également à partir des 4663 "lactations contemporaines " enregistrées dans les mêmes étables et sur la même période de I I années.

\section{a) Vaches contemporaines}

I) $3070 \mathrm{~kg}$ de lait en première lactation, la production laitière atteint un maximum de $445^{\circ} \mathrm{kg}$ de lait en cinquième lactation ; l'accroissement est en moyenne de $574 \mathrm{~kg}$ de lait de la première à la deuxième lactation, de $409 \mathrm{~kg}$ de la deuxième à la troisième, de $237 \mathrm{~kg}$ de la troisième à la quatrième et de $\mathrm{I} 60 \mathrm{~kg}$ de la quatrième à la cinquième (tabl. 3). Chacune de ces performances correspond à la moyenne simple des moyennes obtenues annuellement pour les lactations d'ordre I à 5 : cette façon 
de faire élimine les variations systématiques dues notamment à l'amélioration des conditions de milieu.

\section{TABIEAU 3}

Production laitière en fonction du numéro de lactation

pour les deux catégories de vaches appartenant aux 99 étables ( $\mathrm{kg}$ de lait)

\begin{tabular}{|c|c|c|c|c|c|c|}
\hline \multicolumn{2}{|r|}{ No de lactation } & 1 & $\cong$ & 3 & $4_{4}$ & 5 \\
\hline \multirow{3}{*}{$\begin{array}{l}119.9 \text { vaches } \\
\text { avec } \\
5 \text { lactations } \\
\text { consécutives }\end{array}$} & Moyenne réelle par lactation & 3090 & 3772 & 4215 & 4532 & 4692 \\
\hline & Moyenne corrigée (1) ...... & 3090 & 37292 & 4128 & 4403 & 4510 \\
\hline & Accroissements successifs ... & & 632 & 406 & 275 & 107 \\
\hline \multirow{3}{*}{$\begin{array}{l}\text { Vaches } \\
\text { contem- } \\
\text { poraines }\end{array}$} & $\begin{array}{l}\text { Moyenne simple des moyen- } \\
\text { nes annuelles ............. }\end{array}$ & 3070 & $364^{\prime} 4$ & ${ }^{\prime} 053$ & 4890 & $4450\left({ }^{2}\right)$ \\
\hline & Accroissements successifs ... & & 574 & 409 & 237 & 160 \\
\hline & Effectif............... & 1312 & 1158 & $9 \div 6$ & $71: 3$ & $55 x^{\prime}$ \\
\hline
\end{tabular}

(1) Après élimination des effets dus à l'amélioration continue des conditions de milieu.

(2) Pour les 232 lactations d'ordre 6, la production moyenne était de $4510 \mathrm{~kg}$ de lait.

\section{b) Vaches avec cinq lactations consécutives}

La production des 169 vaches passe alors de $3090 \mathrm{~kg}$ de lait en première lactation à $4602 \mathrm{~kg}$ en cinquième. Cette dernière moyenne inclut en effet la part due à l'amélioration continue des conditions de milieu, puisque, quelle que soit la cohorte considérée, la cinquième lactation est toujours obtenue quatre ans après la première. En appliquant les corrections du paragraphe I $a$, on constate alors que les performances moyennes des vaches contrôlées pendant 5 ans sont à peu près identiques à celles de leurs contemporaines. Ces animaux ne constituent donc pas un échantillon exceptionnel comme on avait tendance à le croire, mais peuvent au contraire servir de référence en vue de l'étude du facteur âge. D'après cet échantillon on observe une bonne répétabilité des performances : 0,52 pour la quantité de lait par lactation, contre 0,69 pour le taux butyreux moyen pondéré $\left({ }^{1}\right)$.

\section{c) Maximum théorique}

L'accroissement de production laitière avec l'âge est, comme l'a montré BRODY (I945), un phénomène comparable à la croissance pondérale dont l'équation est de la forme $\mathrm{A} e^{-k \mathrm{t}}$. I) ans cette équation, le paramètre $\mathrm{A}$ représente le maximum théorique pouvant être atteint par la population considérée dans un milieu déterminé. En utilisant la méthode de W. LIONEI, le maximum théorique, calculé à partir des moyennes corrigées se situe à $4700 \mathrm{~kg}$ : production qui est encore éloignée du maximum observé. Parmi les 169 vaches contrôlées en cinquième

(1) Pour les 5 premières lactations des 169 vaches, le taux butyreux moyen pondéré était respectivement de : $37,27 \mathrm{~g}-36,75 \mathrm{gr}-36,63 \mathrm{~g}-36,5 \mathrm{I}$ et $36,20 \mathrm{~g} \mathrm{p}$. $1000 \mathrm{~g}$. (cf également : AURIOL et GROSClaUde, 1960). 
lactation, 55 p. Ioo n'ont pas eu de sixième lactation. Le fait d'avoir tenu compte des cinquièmes lactations de ces vaches peut avoir entraîné une sous-estimation; BAKELS et GAII (I96I) ont en effet montré que la lactation précédant la réforme ne devait pas être prise en considération dans le calcul du niveau de production en fonction de l'âge, car elle était probablement influencée par les causes qui conduisent à cette réforme.

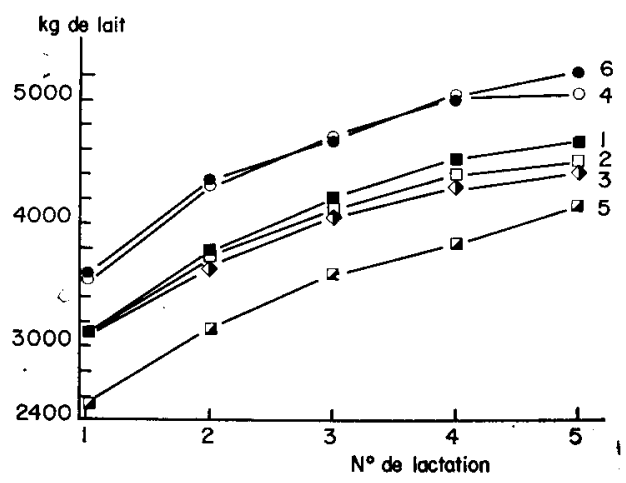

GRAPi.. 2. - Évolution de la production laitière en fonction du numéro de lactation et du niz'eau d'étable.

I : 169 vaches Montbéliardes du département du Jura avec

5 lactations consécutives $\ldots \ldots \ldots \ldots \ldots \ldots \ldots \ldots \ldots \ldots \ldots \ldots$ sans correction ; pour l'amélio-

2 : I69 vaches Montbéliardes du département du Jura avec

3 : Toutes les lactations contemporaines des commensales.

4 : Vaches avec cinq lactations appartenant à des étables de haut niveau.

5 : Vaches avec cinq lactations appartenant à des étables de bas niveau.

Les productions des vaches appartenant à des étables de niveau moyen se confondent avec celles de la courbe $\mathbf{~}$.

6 : 85 vaches Montbéliardes du département du Doubs possédant 5 lactations consécutives.

\section{3. - INFLUENCE DU NIVEAU DE PRODUCTION INDIVIDUEI.}

Si l'augmentation de production $\mathrm{D}_{5-1}$ est en corrélation négative avec le niveau de la première lactation, elle est en corrélation positive avec la somme des cinq lactations. Or, ce dernier paramètre est certainement celui qui traduit le mieux le potentiel laitier d'un animal, puisque les performances initiales peuvent être limitées en partie par des besoins importants, et un appétit réduit. Par contre, les accidents (dont les risques augmentent avec l'âge) sont susceptibles d'empêcher certaines vaches d'atteindre normalement leur production maximum. C'est ainsi que sur les 169 vaches contrôlées, 5 p. Ioo présentaient un maximum en deuxième lactation, I5 p. Ioo en troisième, $39 \mathrm{p}$. Ioo en quatrième et $45 \mathrm{p}$. Ioo seulement en cinquième. Par suite de ces évolutions différentes, l'estimation de la production laitière obtenue en quatrième et cinquième lactation $(z)$ à partir de celle obtenue en première lactation (x) est assez imprécise (équation $I$, tab1. 6).

Le fait que l'augmentation de la production laitière avec l'âge soit plus important pour les animaux à potentiel de production élevé est à rapprocher d'un autre phénomène : l'augmentation plus importante de la production laitière avec l'élévation du niveau d'alimentation pour la même catégorie d'animaux. 


\section{4. - INFLUENCE DU NIVEAU D'ÉtABLE}

\section{a) Production laitière en première lactation et niveau d'étable}

Il existe une corrélation supérieure à $+0,50$ entre le niveau de la première lactation et le niveau d'étable calculé, soit d'après les lactations de génisses, soit d'après "le probit moyen" (tabl. 4). Le " milieu étable " permet donc d'expliquer 25 à $30 \mathrm{p}$. roo de la variance des productions initiales. Ces valeurs sont d'ailleurs comparables à celles rapportées par Hickman et Henderson (1955, 1956). D'autre part, le coefficient de régression du niveau de la première lactation sur le niveau d'étable calculé d'après les lactations de génisses, est de $0,985( \pm 0,135)$. Ce coefficient voisin de $\mathrm{I}, \mathrm{O}$ est peu différent de ceux observés en Angleterre et en Suède (tabl. I) ; il tend à prouver, si l'on admet le raisonnement de ROBERTSON et RENDEL, (1954), que les différences génétiques entre élevages sont pratiquement nulles dans le Jura.

\section{TABLEAU 4}

Corrélations entre les performances des vaches possédant cinq lactations consécutives et les niveaux moyens de production des étables auxquelles elles appartiennent

\begin{tabular}{|c|c|c|c|c|c|c|c|c|c|c|}
\hline \multicolumn{2}{|c|}{$\begin{array}{l}\text { Niveau } \\
\text { d'étable }\end{array}$} & \multicolumn{7}{|c|}{ Vaches avec 5 lactations consécutives } & & \\
\hline \multirow[t]{2}{*}{$\begin{array}{c}\mathrm{Ne} \\
\text { PL1 }\end{array}$} & $\begin{array}{l}\text { Probit } \\
\text { moyen }\end{array}$ & PL1 & PL2 & PL3 & PL4 & PL5 & $D_{5-1}$ & $\begin{array}{c}\text { Age } \\
1^{\text {er vêlage }}\end{array}$ & & \\
\hline & $0,81^{* *}$ & $0,50^{* *}$ & & & & & 0 & & Ne PL1 & \multirow{5}{*}{$\begin{array}{c}\text { Niveau } \\
\text { d'étable } \\
(\mathrm{Ne})\end{array}$} \\
\hline $0,74^{* *}$ & $0,89^{* *}$ & & $0,58^{* *}$ & & & & 0 & & $\mathrm{Ne}$ PL2 & \\
\hline $0,66^{* *}$ & $0,92 * *$ & & & $0,63^{* *}$ & & & & & $\mathrm{Ne}$ PL3,4 & \\
\hline \multirow[t]{8}{*}{$0,50^{* *}$} & $0,83^{* *}$ & $0,43^{* *}$ & & & & $0,46^{* *}$ & $0,28^{*}$ & & Ne PL5 & \\
\hline & & $0,54 * *$ & & $0,60^{* *}$ & $0,56^{* *}$ & $0,41^{* *}$ & 0,18 & & probit moyen & \\
\hline & & & $0,66^{* *}$ & $0,53^{* *}$ & $0,48^{* *}$ & $0,29 * *$ & $-0,34 * *$ & $0,28^{*}$ & PL1 & \multirow{6}{*}{$\begin{array}{c}\text { Vaches } \\
\text { avec } \\
5 \text { lact. } \\
\text { cons. }\end{array}$} \\
\hline & & & & $0,66^{* *}$ & $0,54^{* *}$ & $0,39 * *$ & 0 & 0,19 & PL2 & \\
\hline & & & & & $0,62^{* *}$ & $0,49 * *$ & & & PL3 & \\
\hline & & & & & & $0,58^{* *}$ & \multirow{2}{*}{$0,61 * *$} & & PL4 & \\
\hline & & & & & & & & 0,10 & PL5 & \\
\hline & & & & & & & $0,23^{*}$ & & PL 1 à 5 & \\
\hline
\end{tabular}

Les corrélations comprises entre $\pm 0,10$ sont considérées comme nulles.

** : significatif au seuil $\mathrm{P}=0,01$

* : significatif au seuil $\mathrm{P}=0,05$.

$\mathrm{D}_{5-1}$ : différence de production entre la moyenne des lactations 4 et 5 et la lactation 1.

Probit moyen d'étable : moyenne pondérée des 4 probits correspondants aux 4 moyennes de production calculées pour chaque étable à partir des lactations $1,2,3-4,5$.

$\mathrm{PLi}$ : production laitière de la lactation i. 


\section{b) Accroissement de production laitière avec l'âge et niveau d'étable}

L'estimation de la production laitière adulte $(z)$ d'après le niveau d'étable $(y)$ est donné par l'équation 2 du tableau 6 .

Le paramètre $\mathrm{D}_{5-1}$ est indépendant du niveau d'étable calculé sur les deux premières lactations. Il présente une corrélation positive peu significative avec la moyenne d'étable lorsque celle-ci est calculée, soit sur les cinquièmes lactations, soit d'après le probit moyen.

Dans le tableau 5, nous avons présenté l'évolution de la production laitière des vaches possédant cinq lactations, en les classant dans trois groupes d'élevage : haut, moyen et bas niveau ( ${ }^{1}$ ). Pour les trois groupes, l'accroissement de production est à peu près le même, plus important cependant de la première à la deuxième lactation et plus faible de la troisième à la cinquième, pour les vaches appartenant aux bonnes étables (graph. 2). Il est intéressant de constater que les performances moyennes des vaches appartenant au groupe " haut niveau " correspondent parfaitement à celles entegistrées dans le Doubs pour les vaches possédant également cinq lactations consécutives (données non publiées). Si l'on estime que les Montbéliardes du Doubs, bien que placées dans de meilleures conditions d'élevage, ne sont génétiquement pas différentes de celles du Jura, cela signifie bien que les effets du milieu se répercutent de façon identique sur les performances des différentes lactations.

En supposant que les animaux aient été placés dans d'aussi bonnes conditions au cours des quatre premières lactations qu'en cinquième lactation, les maxima théoriques $A_{1}, A_{2}, A_{3}$, correspondants aux trois niveaux d'étable sont respectivement de 4600,4900 et $5200 \mathrm{~kg}$ de lait.

\section{TABLFAU 5}

Production laitière des vaches Montbéliardes du Jura dans trois classes d'ílevage

\begin{tabular}{|c|c|c|c|c|c|c|}
\hline & \multirow{2}{*}{$\begin{array}{l}\text { Niveau d'étable }\left({ }^{*}\right) \\
\text { (Nbre d'étables) }\end{array}$} & \multicolumn{5}{|c|}{ No de lactation } \\
\hline & & 1 & 2 & 3 & 4 & 5 \\
\hline \multirow{3}{*}{$\begin{array}{c}\text { Vaches avec } 5 \text { lac- } \\
\text { tations consécul- } \\
\text { tives }\end{array}$} & haut (26) & $354 t i$ & 4312 & 4723 & $50 \div 9$ & $507_{t}^{\prime}$ \\
\hline & moyen $\left({ }^{\prime} 1\right)$ & 3063 & 3728 & $4 \div 00$ & 4551 & 4710 \\
\hline & bas $\quad(32)$ & 2557 & 3143 & 3576 & 3843 & 4159 \\
\hline \multirow{3}{*}{$\begin{array}{l}\text { Vaches contempo- } \\
\text { raines }\end{array}$} & haut $\quad(20)$ & 3415 & $\therefore 174$ & \multicolumn{2}{|c|}{4618} & $492 x^{\prime}$ \\
\hline & moyen (41) & $300^{\prime}$ & 3638 & \multicolumn{2}{|c|}{4145} & 14 1'tít \\
\hline & bas $\quad(32)$ & $276 \%$ & 3200 & \multicolumn{2}{|c|}{$369 \%$} & 3988 \\
\hline
\end{tabular}

(*) Haut niveau : probit supérieur à 5,13 (54 vaches).

Niveau moyen : probit compris entre 4,57 et 5,13 (74 vaches).

Bas niveau : probit inférieur à 4,57 ( 41 vaches).

(1) Le taux butyreux moyen pondéré de toutes les vaches appartenant aux étables à haut, moyen et bas niveau de production laitière, était respectivement de $36,5 \mathrm{~g}-36,8 \mathrm{~g}$ et $37, \mathrm{Ig}$ p. $1000 \mathrm{~g}$. 


\section{c) Age au premier vêlage et niveau d'étable}

Il existe une corrélation positive entre l'âge au premier vêlage et le niveau de production laitière $(+0,28)$. En fait, comme nous l'avions déjà observé (AURIOL et RICORDEAC, I958) la régression de la production laitière sur l'âge n'est pas linéaire. En classant les génisses dans les trois catégories d'étables, nous n'avons pas constaté d'influence du niveau d'étable sur l'accroissement de la production en fonction de 1'âge au premier vêlage, ce qui confirme les résultats d'HofMEYr (I955). Il est donc préférable d'utiliser également des facteurs additifs de correction pour l'âge au premier vêlage.

\section{5. - Ixfluexce du niveau de production inttial et du Niveau d'F́table}

\section{Discussion}

La production laitière d'une vache adulte $(z)$ apparait donc comme une fonction de sa performance en première lactation $(x)$ et du niveau moyen de production de son étable $(y)$. Pour essayer de séparer les influences respectives de $x$ et $y$ dans la variation de $z$, il nous faut étudier la régression de $z$ sur chaque couple $(x, y)$. A partir du modèle linéaire à deux variables (équation 3 du tableau 6), nous avons déterminé la droite de régression estimée en tenant compte du niveau d'étable calculé sur la moyenne des lactations 3 et 4 (cette moyenne de production est étroitement liée au probit moyen et a l'avantage de ne pas comprendre les lactations initiales).

\section{TABLEAU 6}

Estimation de la production laitière " adulte " en fonction du nivean de la première lactation et du niveau d'étable.

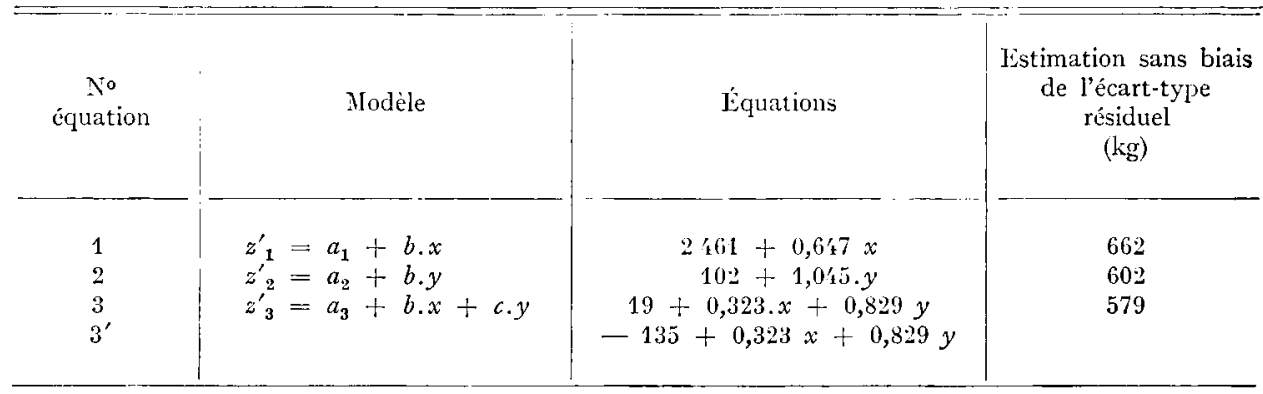

$x$ : première lactation : durée de lactation $\leqslant 300$ jours; âge au vêlage ramené à 32 mois.

$y$ : niveau d'étable estimé par la moyenne des lactations 3 et 4 .

$z$ : moyenne des lactations 4 et 5 : durée de lactation $\leqslant 300$ jours.

L'équation $3^{\prime}$ annule les effets dus à l'amélioration des conditions de milieu.

a) Des trois équations du tableau 6 , la troisième est la plus précise puisqu'elle permet de prévoir la performance adulte $(z)$ à partir du niveau de première lactation $(x)$ et du niveau d'étable $(y)$, avec une erreur, exprimée par l'écart-type résiduel, de $580 \mathrm{~kg}$ ( $12 \mathrm{p}$. Ioo en valeur relative). Nous pouvons dire en conclusion, que l'ac- 
croissement de production laitière avec l'âge est indépendant du niveau d'étable, mais qu'à l'intérieur d'étables de même niveau, cet accroissement est d'autant plus faible que le niveau de production initial (première lactation) est plus éleré. Par exemple, la génisse qui produira $4000 \mathrm{~kg}$ de lait à 32 mois, aura peu de chances de donner une production très supérieure en quatrième et cinquième lactation si la moyenne de ses contemporaines de la même étable est inférieure à $4000 \mathrm{~kg}$. Au contraire, dans une étable dont le niveau moyen est de 6 ooo $\mathrm{kg}$, cette génisse aura la " possibilité " d'accroître ses performances au cours des lactations suivantes.

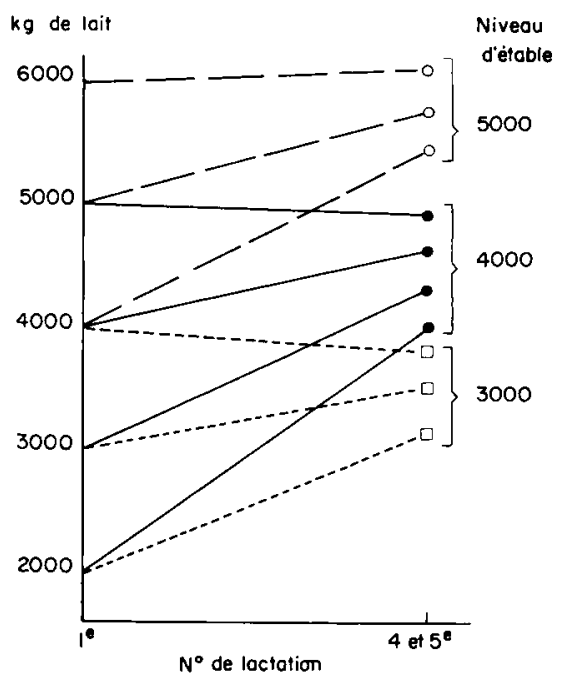

GRAPIr. 3. - Variation de la production laitière avec l'àge, en fonction du niveau de production en première laclation et du niveau d'étable calculé d'après les lactations 3 el 4.

D'après les tables de production établies par GaIness en 1943, il est intéressant de constater que l'augmentation de production laitière avec l'âge est au moins aussi importante, pour les vaches traites trois fois que pour celles traites deux fois par jour. En comparant les performances des "troupeaux de montagne » et des " troupeaux de plaine " d'après les perfornances globales dı contrôle laitier en Suisse, en Allemagne et en Autriche, on constate également, qu'en dépit d'une différence annuelle d'environ I ooo $\mathrm{kg}$ de lait, l'accroissement absolı de production laitière avec l'âge est du même ordre pour les deux catégories de troupeaux. Les conditions d'élevage représentent donc bien un facteur limitant la production laitière. On peut penser que c'est dans les meilleures étables que les vaches sont capables d'atteindre de fortes productions et que dans les troupeaux de même niveau, ce sont les génisses les plus productives au premier vêlage qui manifestent le plus faible accroissement de production avec l'âge, par suite du fait que les apports alimentaires limitent leurs productions ultérieures.

b) Cet échantillon de $I 69$ vaches est réduit, mais non exceptionnel du point de vue longévité et production laitière :

- les animaux de race Montbéliarde sont réputés pour leur longévité $\left.{ }^{(1}\right)$

(') Dans la plupart des concours, une section a longévité, fécondité et rusticité " rassemble les vaches de plus de dix ans, ayant produit sept veaux nés viables en sept vêlages différents. 
puisque parmi les vaches soumises au contrôle laitier, près de 30 p. Ioo ont accompli plus de quatre lactations,

- les vaches ainsi conservées ne sont pas uniquement de bonnes laitières, puisqu'une enquête réalisée dans le Jura a permis de constater que les éleveurs tenaient largement compte de l'aptitude des vaches à vêler régulièrement et à bien se laisser traire. Les observations de BAKFLS (1959) montrent par ailleurs que la durée de vie des vaches est d'autant plus longue que leur niveau de production initial est plus faible.

c) En vue de l'interprétation des résultats concernant la descendance des taureaux d'I. A., l'application (dans les conditions d'élevage du Jura) de la méthode de comparaison des filles avec leurs commensales de même âge pose des problèmes, par suite des faibles effectifs par troupeau. Le fait que les différences génétiques entre troupeaux semblent être faibles autorise le regroupement des génisses dans un petit nombre de classes, en fonction des productions moyennes des élevages où elles sont exploitées ; cette solution ne doit cependant pas être considérée comme définitive et rigoureuse, étant donné que l'utilisation de " taureaux testés " permet maintenant à certains éleveurs, de constituer de véritables familles dont la valeur d'élevage est nettement supérieure à la moyenne. Puisque l'effet de l'âge sur la production laitière est indépendant de la moyenne d'étable, on peut calculer cette movenne d'étable à partir cle toutes les lactations disponibles, en ajustant la distribution des âges observée à une distribution représentative de la moyenne de la population, ou ce qui revient au même, en utilisant la méthode décrite par STLENBERG (1955).

La transformation des productions en variable "probit ", après ventilation des données par année, âge et numéro de lactation, intervalle et saison de vêlage, semble cependant préférable du point de vue calcul et interprétation comme nous avons pu le constater dans d'autres domaines (contrôle de la croissance et de la conformation en races Charolaise, Normande et Montbéliarde, LEFEBVRE, AURIol et DUMONT).

Le niveau de production par étable ne constitue en fait qu'une donnée globale. Pour obtenir le maximum d'informations, il serait utile de déterminer, parmi les principales sources de variation (choix des reproducteurs, alimentation, techniques d'élevage, conditions de traite, pratique des réformes, état sanitaire) celles qui expliquent le mieux les différences entre élevages.

Reçu pour publication en novembre 1963 .

\section{SUMMARY}

INFLUENCE OF LACTATION NUMBER AND HERD PRODUCTION-LEVEL ON MILK YIELD OF " MONTBÉLIARDE 》 COWS

The object of this study is to determine the influence of different factors - year, level of initial performances, herd production level - on the evolution of milk production with age.

During the period 1947 to $195^{8}$, we retained in 99 herds of the Jura region, 169 Montbéliardes cows having five consecutive lactations ( $\mathrm{I}-5)$ and a total of 4663 " contemporary cow " records from the same herds. For each of the herds, 4 production averages and a probit average were calculated. 
I) From 1947 to $195^{8}$, a continual increase of the average milk production, mainly due to the amelioration of environmental conditions, was registred : $30 \mathrm{~kg}$ per year for the first lactations and $45 \mathrm{~kg}$ for the lactations 2 to 5 (graph. I).

2) The production increase actually due to the influence of age, is equal for the sample of I69 cows with five consecutive records and their contemporaries in the same herds, when eliminating the effect of the continuous amelioration of the environmental conditions. This increase is in average, $600 \mathrm{~kg}$ of milk from the first to the second lactation, $410 \mathrm{~kg}$ from the second to the third, $260 \mathrm{~kg}$ from the third to the fourth and $130 \mathrm{~kg}$ from the fourth to the fifth (table 3 ). The repeatability coefficients calculated through the records of the 169 cows with five lactations are 0.52 for milk yield and 0.69 for butterfat percentage.

3) The "herd environment " explains 25 to $30 \mathrm{p}$. I00 of the variance of the first lactations. The regression coefficient of the production level of cows in first lactation to their herd's average is 0.98 .

4) The average milk production of fourth and fifth lactations was estimated from (table 6) :

- first lactation production level (equation I);

- herd's average (equation 2);

-- both these two variables (equation 3 ).

However, with the third equation, the standard deviation of the errors between estimated and true adult production is still $579 \mathrm{~kg}$ of milk.

The absolute value of milk production increase with age is independant of the herd's production level, but within herds of the same level this increase is in negative relationship with the level of production during the first lactation.

5) In the discussion, the regrouping of herds in a small number of classes is considered, to estimate the "breeding value " of young bulls through testing by the method of " contemporary comparisons".

\section{RÉFÉRENCES BIBLIOGRAPHIQUES}

Aurioc P., 1955. Influence du mois de vêlage sur la production laitière des vaches Pie Rouge de lest dans le Jura. Ann. Zootech., 4, i89-200.

Auriol P., Ricordeau G., I958. In a Récentes études sur divers aspects du problème de la production du lait. Ann. Nutr., Paris, 12 , I5-17.

Auriol P., Grosclaude F., Ig6o. Recherches sur jumeaux bovins. I. Production et développement corporel des vaches laitières en fonction de l'âge au prémier vêlage. Résultats préliminaires. Ann. Zootech., 9, $301-343$.

Auriol P., Grosclaude F., 1960. Évolution, avec l'âge, de la composition du lait de vaches. Ann. Zootech., 9 , I $21-\mathrm{I} 32$.

BAKELS $F$., I959. Untersuchungen über die beziehungen zwischen milchleistung und nutzungsdauer in einer Allgäuer herde. Arb. Inst. Tierz. Vererb. u. KonstForsch. Univ. München., 1, 1-3o.

BAKELS F., GaLL C., 196r. Ein beitrag zur berechnung durchschnittlicher Jahresmilchleistungen von Altersklassen beim Kind in Herdbuchpopulationen. Zuchttngskunde, 34, 6-I4.

Brody S., I945. Bioernergetics and growth. Reinhold Publishing Corporation, U. S. A.

Brumby P. J., I960. Twin research at Ruakura, New Zealand, Proc. VII study meeting F. E. Z. Stockhulm, 99-105.

BRUmby P. J., 196r. The causes of differences in production between dairy herds. Anim. Prod., 3, 277-294.

DIETRICI H., 1957. Untersuchungen über die Lebensleistung schwarzbunter Niederungsrinder in Sachsen - Anhalt unter besonderer Berücksichtigung der Milchmenge, des prozentischen Fettgehaltes sowies des Alters. Kühn-Archi., 71, $155^{-210}$.

Gaines W. L., 1943. An analysis of milking Shorthorn milk records. Univ. Illinois. Agric. Exp. St Bull., 498, $55^{1-576 .}$

Gaunt S. N., BARTLETt M. A. G., 1958. Influence of herd and stablemate averages on production records of artificially sired (A. I.) progeny. J. Dairy Sci. Abstr., 41, 341.

Hickma. C. G., IIENderson C. R., 1955. Components of the relationship between level of production and rate of maturity in dairy cattle. J. Dairy Sci., 38, $883-890$.

Henderson C. R., 1956. Cornell Research on methods of selecting dairy sires. Proc. N. Z. Soc. Anim. Prod., 16, 69-74.

HOFMEYR J., I955. A study of Danish and Swedish progeny testing methods for dairy bulls. Kgl. Lantbr. Ann. Sverige, 22, 425-488.

Johansson I., Hansson A., I940. Causes of variations in milk and butterfat yield for dairy cows. Kgl. Lantbr. Akad. Tidskr., 79, I-1 27.

Johansson I., Robertson A., 1952. Progeny testing in the breeding of farm animals. Proc. Brit. Soc Anim. Prod., 79-105. 
I.efebvre J., 1946. Étude de l'hérédité des aptitudes laitières et beurrières chez les bovins de race Normande. Ann. Agron., 4, 492-510.

N. A. T., I960. Production laitière des vaches Suédoises en fonction du mois de vêlage. Svensk IIusdjursskötsel., 6-7, 190-195.

Pirchner F., 1959. Statistisch-genetische Untersuchugen an alpinem Braunvieh. Z. Tierz. Zïchtbiol., 73, $129^{-1} 50$.

Pirchner F., Lush J. L., 1959. Genetic and environmental portions of the variation among herds in butterfat production. J. Dairy Sci., 42, i $15-\mathrm{I} 22$.

Poly J., I958. Dans " Récentes études sur divers aspects du problème de la production du lait ". Ann. Nutr., Paris, 12, 8-14.

RoBertson A., Rendel J. M., I954. The performance of heifers got by Artificial Insemination. J. Agric. Sci., 44, I84-190.

Robertson A., McArtiur A. T. G., 1955. Genetic differences between bull-breeding herds. Proc. Brit. Soc. Anim. Prod., 94, Iо I-108.

Robertson A., Khishis S. S., i958. The effect of selection for heifer milk yield on the production level of mature cow. J. Agric. Sci., 50, I 2-I 5 .

SANDERS H. G., I928. The variations in milk yields caused by season of the year, service, age and dry period, and their elimination. Part III. The age. J. Agric. Sci., 18, 46-67.

SFARLE S. R., Henderson C. R., I959. Etablishing age-correction factors related to the level of herd production. J. Dairy Sci., 42, 824-835.

Searle S. R., Henderson C. R., I960. Judging the effectiveness of age-correction factors. J. Dairy Sci., 43, 966-974.

SEarle S. R., 1962. Age and herd effects in New Zealand dairy cow records. J. Dairy Sci., 45, 82-85.

SCIINDLER II., 1959. Genetic and environmental differences between herds as a factor in sire proving. Proc. Israel. Soc. Appl. Anim. Genet, 1959, 31-36.

Steenberg If. J., I955. A new method of analysing herd averages. Milk Rec. Res. Res. Anim Husb Dairying $S . A / r \mathrm{n}^{\circ} \mathrm{I}, \mathrm{I} 3 \mathrm{I}^{\mathrm{I}} \mathrm{I} 33$.

Syrstad O., I960. Progeny testing of dairy sires. Meld. Norg. LandbrHögsk., 39, I-34.

Thompson N. R., BALDwin V. L., GraF G. C., I96r. Genetic and environmental differences among dairy herds (Abstr.). J. Dairy Sci., 44, 980 98I.

VAN Vleck L. D., Henderson C. R., r96r. Ratio factors for adjusting monthly test-day data for age and season of calving and ratio factors for extending part lactation records. J. Dairy Sci., 44, I093-1 I02.

WhRD A. H., CAmpbell J. T., 1938. Practical applications of Age Conversion factors Relating to dairy Cattle Production (butterfat) records. J. Agric. Sci., 38, 509 .

Wiener G., 1960. Factors influencing average milk yield of herds at two levels of production. Anim. Prod., 2, II7-I zo.

WINTERS W. R., ERB R. E., 1957. A progress report on the use of contemporary herd average in sire evaluation. Proc. W. Div. Amer. Dairy Sci. Ass., 38th Ann. Meet., 1957, I 40-160. 